\title{
Paediatric Investigators Collaborative Network on Infections in Canada (PICNIC) study of the current landscape of invasive meningococcal disease in children
}

\author{
Joan L Robinson ${ }^{1 *}$, Sergio Fanella², Alison Lopez², Craig Frankel ${ }^{3}$, Jane McDonald ${ }^{4}$, \\ Mohammad Alghounaim ${ }^{4}$, Robert Slinger ${ }^{5}$, Jennifer Bowes ${ }^{5}$, Sarah Khan ${ }^{6}$, Jeannette L Comeau ${ }^{7}$, \\ Kirk Leifso ${ }^{8}$, John Gunawan', Michelle Barton ${ }^{3}$
}

\section{Abstract}

Background: Immunizations have led to a decrease in the incidence of invasive meningococcal disease (IMD) in Canada, but this infection still leads to significant morbidity and mortality.

Objectives: The purpose of this study was to determine the burden of illness and management of IMD in paediatric hospitals.

Methods: Data were collected on all cases of IMD in eight paediatric hospitals from 2013 to 2017.

Results: There were 17 cases of IMD. Three of eight hospitals had no cases. Just over half of the cases were serogroup $B(n=9)$; a quarter $(n=4)$ were serogroup $W$; less than a quarter $(n=3)$ were serogroup $Y$; and one was unknown. Two infected children were not started on antibiotics until day one and day five after the initial blood culture was collected, but had uneventful recoveries. Six cases required admission to intensive care units; two died. Six cases had probable or proven meningitis. Thrombocytopenia was documented in seven cases. All cases had elevated C-reactive protein levels. Seven children received more than seven days of antibiotics; of these seven, only two had complications that justified prolonged therapy (subdural empyema and septic knee). Six cases had a central line placed.

Conclusion: IMD is now rare in Canadian children, but about one-third of the cases in our study required treatment in the intensive care unit and two died. Clinicians appear to not always be aware that a five to seven-day course is adequate for uncomplicated cases of bacteremia or meningitis.
This work is licensed under a Creative Commons Attribution 4.0 International License.

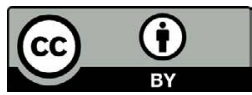

Affiliations

1 Department of Pediatrics, University of Alberta, Edmonton, $A B$

2 Department of Pediatrics, University of Manitoba, Winnipeg, MB

${ }^{3}$ Department of Paediatrics, Western University, London, ON

${ }^{4}$ Department of Pediatrics, McGill University, Montréal, QC

${ }^{5}$ Department of Pediatrics, University of Ottawa, Ottawa, ON

${ }^{6}$ Department of Pediatrics, McMaster University, Hamilton, ON

${ }^{7}$ Department of Pediatrics, Dalhousie University, Halifax, NS

${ }^{8}$ Department of Pediatrics, Queen's University, Kingston, ON

\section{*Correspondence:}

jr3@ualberta.ca

Khan S, Comeau JL, Leifso K, Gunawan J, Barton M. Paediatric Investigators Collaborative Network on Infections in Canada (PICNIC) study of the current landscape of invasive meningococcal disease in children. Can Commun Dis Rep 2020;46(10):339-43. https://doi.org/10.14745/ccdr.v46i10a05

Keywords: immunization, meningococcal disease, IMD, serotype W

\section{Introduction}

Invasive meningococcal disease (IMD) manifests as bacteremia with or without seeding to other sites including the meninges. Despite marked advances in intensive care, IMD still leads to significant morbidity and mortality worldwide, with rates of over 200 cases per 100,000 population in some countries in select years (1).
An increasing number of cases due to a virulent serogroup $C$ strain from the sequence type 11 (ST-11) clonal complex (CC) was first noted in 1986 in Canada, with outbreaks occurring in 1999-2001 (2). In response to these outbreaks, monovalent serogroup $C$ meningococcal vaccines were added to routine infant immunization programs across Canada in 2002 through 2007 (2). All jurisdictions provide one dose at 12 months of 
age and some also provide doses at younger ages (3). One dose of quadrivalent vaccine is now provided to adolescents in Canada with the exception of Québec, where a booster dose of monovalent $C$ vaccine is given (4).

The incidence of IMD in Canada has decreased from approximately 1.5 cases per 100,000 population in 1990-1992 to 0.5 cases per 100,000 in 2006-2011 (5), primarily due to the virtual disappearance of disease due to serogroup C (6).

Up-to-date national data on uptake of infant or adolescent meningococcal vaccines in Canada are not available, but extrapolating from other vaccines, it seems likely that the uptake is approximately $85 \%$ for the infant vaccine and somewhat lower for the adolescent vaccine (7). Vaccine efficacy is difficult to study given the rarity of IMD, but waning of titres (8) and vaccine failures (9) have been described with monovalent $C$ vaccine in other countries. To date, there is no evidence that serotype replacement has occurred with meningococcal vaccines in Canada $(3,6)$ or elsewhere $(10)$.

National data on IMD have appeared in peer review publications since 2011 (5). The objective of this study was to describe the characteristics, management and outcomes of children with IMD in paediatric tertiary care hospitals in Canada in recent years.

\section{Methods}

This retrospective chart review study was conducted by the Paediatric Investigators Collaborative Network on Infections in Canada (PICNIC) at eight participating tertiary care paediatric hospitals in Halifax, Montréal, Ottawa, Kingston, Hamilton, London, Winnipeg and Edmonton. IMD was defined as a positive blood culture for Neisseria meningitidis. At each of the sites, the local laboratory identified inpatients and outpatients, aged up to and including 17 years, with positive blood cultures for $N$. meningitidis, from January 1, 2013, through December 31, 2017. Cases of bacteremia with positive blood cultures for species other than N. meningitidis were excluded. There were no other exclusions.

Chart reviews were then conducted for cases identified by the laboratories. Data on demographics, clinical course, treatment and outcome for eligible cases were entered into REDCap (Research Electronic Data Capture), a secure web application for building and managing online surveys and databases hosted by the University of Alberta. Given the small sample size, data analysis was limited to descriptive statistics.

Approval to conduct the study was obtained from the ethics review board at each site.

\section{Results}

\section{Characteristics of cases of invasive meningococcal disease}

From 2013 through 2017, five of the eight participating sites reported 17 cases of $N$. meningitidis bacteremia, with four cases reported annually in 2013 and 2014, and three cases annually in 2015,2016 and 2017. The remaining three sites had no cases of bacteremia due to $N$. meningitidis.

Five cases of bacteremia due to other Neisseria species were excluded: two cases of $N$. sicca, one each of $N$. mucosa and $N$. gonorrhea and one of an unidentified species. Just over half of the cases $(n=9)$ were serogroup $B$; a quarter $(n=4)$ were serogroup $W$; less than a quarter $(n=3)$ were serogroup $Y$; and one was an unknown serogroup (Figure 1).

Figure 1: Age and serogroup of cases of invasive meningococcal disease $(n=17)$ at eight tertiary care paediatric hospitals in urban centres, Canada, 2013-2017

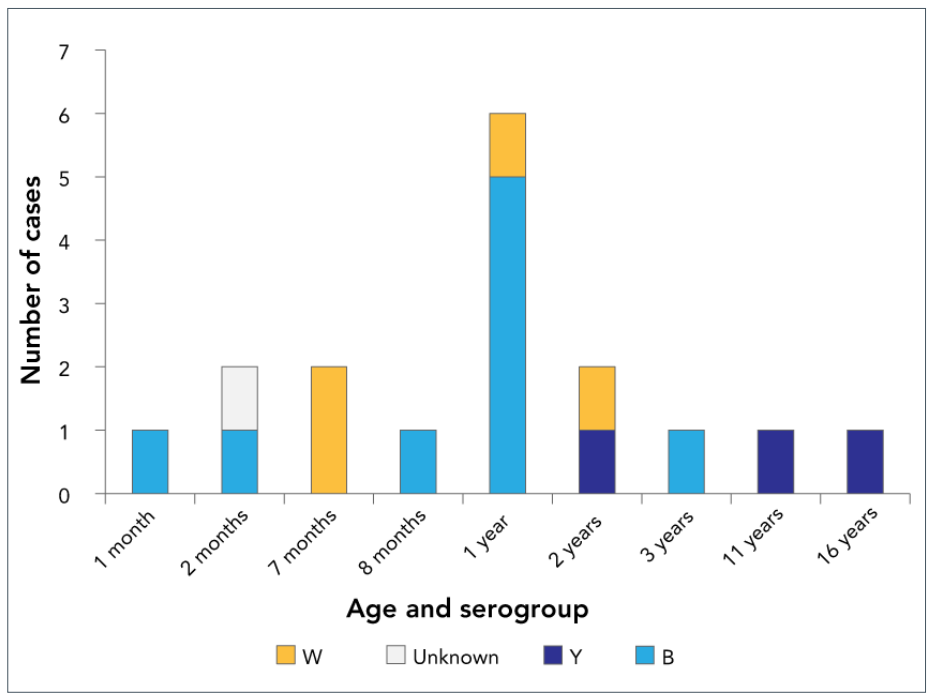

Of the 17 children, 13 were boys and six were younger than 12 months (Figure 1). Two cases had major underlying conditions: a two-month-old had repaired gastroschisis and a one-year-old had intestinal failure, neither of which has been linked to IMD. However, a two-year-old case was subsequently diagnosed with complement component 8 deficiency, which does increase the risk of IMD.

\section{Clinical course of cases}

Three patients were transferred to the participating sites from smaller hospitals for unknown reasons. All but two were admitted on the day the blood culture was collected and were started on empiric antibiotic therapy that covered $\mathrm{N}$. meningitidis. A febrile two-month-old patient with repaired gastroschisis was sent home and admitted for antibiotics the following day when the blood culture was found to be growing gram-negative 
bacilli. A three-year-old patient with no history of fever was admitted five days after blood was sampled for culture when growth of $N$. meningitidis was found; the reason for obtaining the blood culture was unknown and the child remained bacteremic at admission. Both cases with delayed treatment had uncomplicated courses.

Data on fever were available for 15 cases. With the exception of the three-year-old mentioned above who was never febrile, all had fever prior to or within 24 hours of admission to hospital. Three children remained febrile for longer than 72 hours after appropriate antibiotics were started; all had probable meningitis.

Six of the 17 cases required admission to intensive care unit (ICU), with four requiring mechanical ventilation. Two of the patients, a 16-year-old with serogroup $Y$ and a one-year-old with serogroup $B$, died on day 5 and day 18 of their ICU stay, respectively; both had extensive necrotic tissue. The four other patients stayed in the ICU for 1, 2, 6 and 22 days. The child with the longest stay required bilateral below-knee amputations.

\section{Laboratory findings}

It is not known how many of the 17 children had lumbar punctures for a definitive diagnosis of meningitis, but two cases were proven meningitis and four were probable meningitis. The two proven cases, with $N$. meningitidis identified in cerebrospinal fluid (CSF), were a two-month-old and a two-year-old.

The first two probable cases were a two-month-old and seven-month-old with sterile CSF with pleocytosis (obtained after antibiotics); the seven-month-old developed a subdural empyema. The other two probable cases were the 16-year-old who died and a one-year-old who presented with a febrile seizure and was sent home on amoxicillin three days prior to the positive blood culture being drawn; neither had a lumbar puncture performed. Other sites of infection included septic arthritis of the knee in a two-year-old and circumferential cellulitis of the arm that was initially thought to be compartment syndrome in a one-month-old.

Initial peripheral white blood cell count ranged from 2.0 to 36.9 $\times 10^{\circ} / \mathrm{L}$ (mean $17.1 \times 10^{9} / \mathrm{L}$ ), with peak white blood cell count ranging from 13.7 to $40.9 \times 10^{9} / \mathrm{L}$ (mean $24.3 \times 10^{9} / \mathrm{L}$ ). Seven children had thrombocytopenia (<150 × 10\%/L). Mean peak C-reactive protein levels were $121 \mathrm{mg} / \mathrm{L}$ in the nine cases where it was measured (range: $50.7-189.9 \mathrm{mg} / \mathrm{L}$ ).

\section{Antimicrobial therapy}

The median duration of antibiotic therapy for IMD was seven days (minimum five days and maximum 17 days). Seven children received courses of antibiotic therapy that exceeded seven days; only two, the cases of subdural empyema and septic arthritis, clearly required a course of antibiotics longer than seven days.

Treatment was with ceftriaxone in all but one case; this patient was treated with cefotaxime. Patients were admitted for the entire course of intravenous antibiotics except for one who was treated on their sixth (and final) day as an outpatient. Oral antibiotics were used only for the child with septic arthritis who received seven days of amoxicillin-clavulanate treatment following eight days of ceftriaxone.

Ten patients were managed with peripheral intravenous (IV) cannulation only; of the remaining seven cases, one child already had a peripherally inserted central line (PICC) for intestinal failure, three children had traditional central lines placed, two children had PICCs inserted and one patient who was admitted to ICU had more than one central line inserted.

\section{Discussion}

There were 17 cases of IMD in eight Canadian paediatric hospitals over a five-year period. Although there are no data for previous years, from 2013 to 2017 there was less than one case per centre every two years, which is low considering that these are primarily regional referral centres. This success may be attributed to infant and adolescent immunization programs. Still, about one-third of the children required ICU admission and two died.

Although this is a small number of cases, a hypervirulent clone that is not well covered by the current vaccines could lead to a much larger outbreak. For example, there are concerns that the hypervirulent serogroup W ST-11 CC is emerging in Canada (11). Serogroup W accounted for $19 \%$ (15/80 cases) of all cases of IMD in 2016 (11) versus $24 \%$ of cases in the current study. The ST-11 CC was first detected in Canada in 2014, but accounted for 85 of 93 serogroup W cases (91\%) in 2016-2018 (11). This clone appears to primarily infect adults; it is possible that adolescent immunization has prevented disease in immunized children and indirectly prevented disease in younger siblings by preventing carriage.

The most common serogroup in IMD in children in Canada (6) and the United States (10) is B. An infant and an adolescent monovalent $B$ vaccine are now licensed in Canada but are not used routinely due to the large number needed to vaccinate (NNV) to prevent one case of IMD (3). The relatively small absolute number of cases due to serogroup $B$ infection in the current study (nine cases in eight centres over five years) supports that decision. The first dose of vaccine can be given at two months of age (depending on the province), but one dose is probably not sufficient to prevent IMD. Unlike serogroup C vaccines, the serogroup $B$ vaccine licensed for infants does not prevent carriage so widespread use in the population may not prevent disease in young infants (12).

Management of IMD in Canada could be improved. Seven of the 17 cases received more than seven days of antibiotics (a five to seven-day course is recommended for bacteremia or meningitis) (13); only two of the seven cases had an indication for 
longer therapy (subdural empyema and septic arthritis). Clinicians assume that an infection with an approximately $10 \%$ mortality rate (14) warrants a longer course of antibiotics, but this prolongs hospital stay and often necessitates placement of a central line.

\section{Strengths and limitations}

The strength of this study is that it provides data on management and outcomes for IMD in Canada in recent years. The major limitation is that the study is not population based; some of the eight participating sites have no defined catchment area.

In addition, not all Canadian paediatric tertiary care hospitals participated in the study. What's more, children with an uncomplicated course or with rapid demise may never be admitted to a tertiary care hospital. Another limitation is that follow-up data were not collected; long term sequelae are typically not apparent at discharge.

Some of the longer courses of antibiotics may have been prescribed for legitimate indications that were not documented in patients' charts. Other limitations are that only eight centres were involved and immunization status was not available.

\section{Conclusion}

IMD is now a rare condition in Canadian paediatric hospitals, although over one-third of cases identified in eight paediatric hospitals from 2013 to 2017 required ICU admission and two died. Many residents in training may never see a case. In this era of antimicrobial stewardship, clinicians should be reminded that a long course of antibiotics is rarely indicated for IMD. Ongoing surveillance should continue so that outbreaks are rapidly identified. Future studies should verify immunization status.

\section{Authors' statement}

$M B$ and JR had the idea for the study, wrote the protocol and designed the REDCap (Research Electronic Data Capture) form. JR wrote the first draft of the manuscript. All other authors collected data and reviewed the manuscript.

\section{Competing interests}

None.

\section{Funding}

No funding was received for this study.

\section{References}

1. Jafri RZ, Ali A, Messonnier NE, Tevi-Benissan C, Durrheim D, Eskola J, Fermon F, Klugman KP, Ramsay M, Sow S, Zhujun S, Bhutta ZA, Abramson J. Global epidemiology of invasive meningococcal disease. Popul Health Metr 2013 Sep;11(1):17. DOI PubMed
2. De Wals P. Epidemiology and control of meningococcal disease in Canada: a long, complex, and unfinished story. Can J Infect Dis Med Microbiol 2019 Nov;2019:8901847. DOI PubMed

3. Robinson JL. Update on invasive meningococcal vaccination for Canadian children and youth. Paediatr Child Health 2018 Feb;23(1):e1-4. DOI PubMed

4. Public Health Agency of Canada. Provincial and territorial routine and catch-up vaccination schedule for infants and children in Canada. Ottawa (ON): Government of Canada; (modified 2020-07-29; accessed 202007-29). https://www.canada.ca/fr/sante-publique/ services/renseignements-immunisation-provinces-etterritoires/programmes-vaccination-systematique-provin ces-territoires-nourrissons-enfants.html

5. Public Health Agency of Canada. Invasive meningococcal disease. Ottawa (ON): Government of Canada (modified 2019-11-26; accessed 2020-07-29). https://www. canada.ca/en/public-health/services/immunization/ vaccine-preventable-diseases/invasive-meningococcaldisease/health-professionals.html

6. Sadarangani M, Scheifele DW, Halperin SA, Vaudry W, Le Saux N, Tsang R, Bettinger JA, Bettinger JA; investigators of the Canadian Immunization Monitoring Program, ACTive (IMPACT). The impact of the meningococcal serogroup $C$ conjugate vaccine in Canada between 2002 and 2012. Clin Infect Dis 2014 Nov;59(9):1208-15. DOI PubMed

7. Robinson JL. Potential strategies to improve childhood immunization rates in Canada. Paediatr Child Health 2018 Aug;23(5):353-6. DOl PubMed

8. Perrett KP, Richmond PC, Borrow R, Nolan T, McVernon J. Antibody persistence in Australian adolescents following meningococcal C conjugate vaccination. Pediatr Infect Dis J 2015 Mar;34(3):279-85. DOI PubMed

9. Matar R, Hong E, Levy C, Guillot M, Cohen R, Taha MK, Madhi F. Vaccine failure after meningococcal $C$ conjugate vaccine may be linked to decline of bactericidal titers and absence of herd immunity. Pediatr Infect Dis J 2015 Oct;34(10):1142-3. DOI PubMed

10. Centers for Disease Control and Prevention. Meningococcal disease: disease trends. Atlanta (GA): CDC (modified 2019-05-31; accessed 2020-07-29). https://www.cdc.gov/ meningococcal/surveillance/index.html\#trends

11. Tsang RS, Hoang $L$, Tyrrell GJ, Minion J, Van Caeseele $P$ Kus JV, Lefebvre B, Haldane D, Garceau R, German G, Zahariadis G, Hanley B. Increase in ST-11 serogroup W Neisseria meningitidis invasive meningococcal disease in Canada, 2016-2018. Can Commun Dis Rep 2019 Jun;45(6):164-9. DOI PubMed

12. Marshall HS, McMillan M, Koehler AP, Lawrence A, Sullivan TR, MacLennan JM, Maiden MC, Ladhani SN, Ramsay ME, Trotter C, Borrow R, Finn A, Kahler CM, Whelan J, Vadivelu $\mathrm{K}$, Richmond $\mathrm{P}$. Meningococcal $\mathrm{B}$ vaccine and meningococcal carriage in adolescents in Australia. N Engl J Med 2020 Jan;382(4):318-27. DOI PubMed 
13. Meningococcal infections. In: Red book: 2018-2021 Report of the Committee on Infectious Diseases, 31st edition. Kimberlin DW, Brady MT, Jackson MA, Long SS, editors. Ithaca (IL): American Academy of Pediatrics; 2018. p. 550-61.
14. Sadarangani M, Scheifele DW, Halperin SA, Vaudry W, Le Saux N, Tsang R, Bettinger JA; investigators of the Canadian Immunization Monitoring Program, ACTive (IMPACT). Outcomes of invasive meningococcal disease in adults and children in Canada between 2002 and 2011: a prospective cohort study. Clin Infect Dis 2015 Apr;60(8):e27-35. DOI PubMed

\section{PREVENT THE SPREAD OF COVID-19}

CORONAVIRUS DISEASE (COVID-19): HOW TO CARE FOR A PERSON WITH COVID-19 AT HOMEADVICE FOR CAREGIVERS
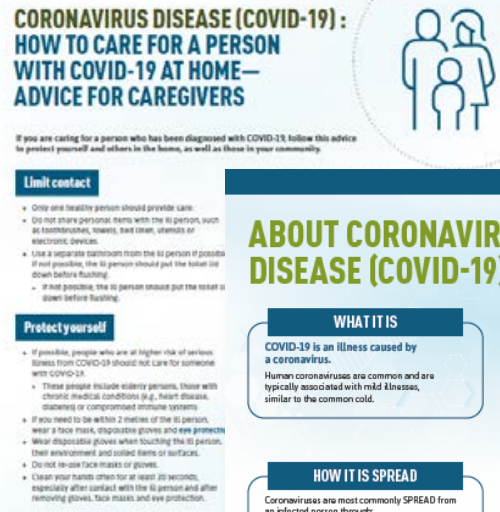

\section{ABOUT CORONAVIRUS DISEASE (COVID-19)}

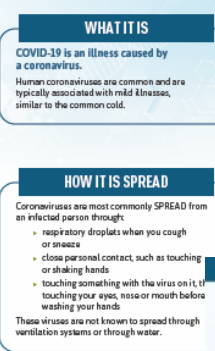

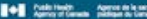

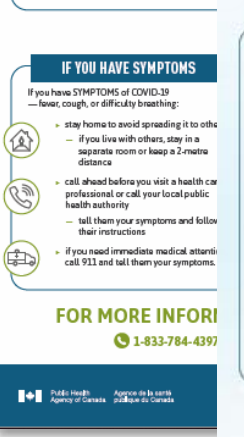

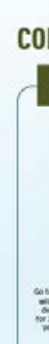
CORONAVIRUS DISEASE (COVID-19)

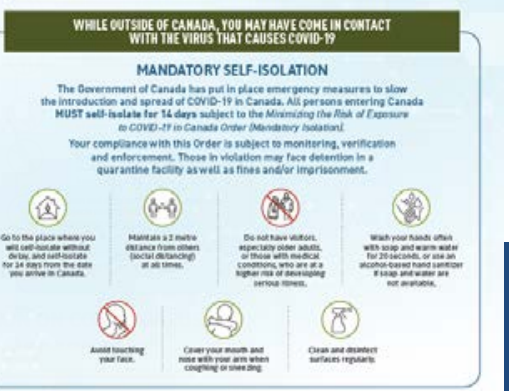

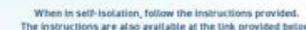

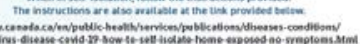
MCWITOR YOUR MGATH FOR L LATS

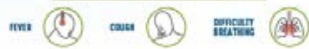

COVID-19

Awareness Resources

- Get the latest guidance and awareness resources

- Some resources are available in multiple languages

\section{Visit} www.canada.ca/coronavirus 